\title{
Construção de Uma Escala de Satisfação no Trabalho para Trabalhadores Informais
} (EST-TI)

\author{
Building a Job Satisfaction Scale for Informal Workers (EST-TI)
}

Yohans De Oliveira Esteves ${ }^{1}$, Ione Vasques-Menezes ${ }^{2}$, Clarissa Pinto Pizarro de Freitas ${ }^{3}$

\section{RESUMO}

O tema satisfação do trabalho tem sido objeto de constante estudo por diversas áreas de conhecimento, em especial, as áreas de Administração, Economia, Sociologia e Psicologia. Com relação ao trabalho informal não existem instrumentos para medir o nível de satisfação. Com base na concepção que aborda a relação trabalho-trabalhador através dos aspectos objetivos e subjetivos do trabalho e considerando a não existência de uma escala para avaliação da satisfação no trabalho voltada para trabalhadores informais e para trabalhadores por conta própria, o objetivo deste estudo é construir e validar a Escala de Satisfação no Trabalho para Trabalhadores Informais (EST-TI). Participaram do estudo 328 trabalhadores informais pertencentes as diversas áreas de atuação. A coleta de dados foi realizada com trabalhadores informais que atuam no Leste Metropolitano do Estado do Rio de Janeiro no período compreendido entre março e outubro de 2017. Desta forma, a discussão e validação de uma escala de satisfação no trabalho voltada especificamente para os trabalhadores informais pode ser bastante útil para realização de diagnósticos e investigações futuras e representa um ganho significativo pois fornecerá aos pesquisadores um instrumento específico para o estudo em trabalhadores informais, com características e itens adequados a este tipo de trabalhador.

Palavras-chave: Escala de Satisfação. Informalidade. Satisfação no Trabalho. Trabalhadores Informais. Trabalho Informal.

\section{ABSTRACT}

The subject of job satisfaction has been the object of constant study by several areas of knowledge, especially in the areas of Administration, Economics, Sociology and Psychology. Regarding informal work, there are no instruments to measure the level of satisfaction. Based on the conception that approaches the work-worker relationship through the objective and subjective aspects of the work and considering the non existence of a scale for evaluation of the satisfaction in the work directed to informal workers and for self-employed, the objective of this study is to construct and to validate the Informal Worker Satisfaction Scale (EST-TI). A total of 328 informal workers from different areas participated. The data collection was carried out with informal workers who work in the Metropolitan East of the State of Rio de Janeiro from March to October 2017. Thus, the discussion and validation of a scale of job satisfaction focused specifically on informal workers can be very useful for conducting future diagnoses and investigations and represents a significant gain as it will provide researchers with a specific instrument for the study of informal workers with characteristics and items appropriate to this type of worker.

Keywords: Informality. Informal Work. Informal Workers. Job Satisfaction. Satisfaction Scale.
1 Administrador, Doutor em Psicologia, UFF.

E-mail: yoesteves@gmail.com

2 Psicóloga, Doutora em

Psicologia, Professora do PPGP da UNIVERSO.

E-mail: vasques-

menezes@gmail.com

3 Psicóloga, Doutora em

Psicologia, Professora do PPGP

da UNIVERSO.

E-mail: freitas.cpp@gmail.com 


\section{INTRODUÇÃOO}

O tema satisfação do trabalho tem sido objeto de constante estudo por diversas áreas de conhecimento. Em especial, as áreas de Administração, Economia, Sociologia e Psicologia (Bacha et al., 2015; Brandão et al., 2014; Marques, Borges \& Reis, 2015; Souza, Milani \& Alexandre, 2015), sendo uma das principais variáveis no âmbito do comportamento organizacional (SIQUEIRA, 2008).

A segunda metade da década de 1930 marca o início do processo de busca pela definição do construto da satisfação e das formas que este afeta dentro e fora das organizações a qualidade de vida dos trabalhadores. Desde então, devido à grande importância para o contexto laboral, a satisfação no trabalho vem sendo estudada das mais variadas formas (CARLOTTO \& CÂMARA, 2008; SIQUEIRA, 1995).

O construto começou a ser estudado por Locke (1976) cuja a definição ainda é uma das mais importantes na literatura. Para Locke (1976), satisfação no trabalho é entendida como um estado positivo ou agradável resultante da avaliação do trabalhador referente as experiências relacionadas ao trabalho. Locke (1969) afirmava que os níveis de satisfação e insatisfação poderiam ser distintos dentro de uma mesma organização em função das diferentes percepções e dos valores atribuídos ao trabalho pelos trabalhadores.

Desta forma, a relação entre o que o trabalhador percebe e o que ele deseja com relação ao trabalho podem afetar os níveis de satisfação e insatisfação. Para Locke existem três níveis de atuação inerentes à satisfação no trabalho: a percepção de aspectos do trabalho; a noção de padrões de valor; e a conexão ou discordâncias entre os dois primeiros níveis (COELHO \& FAIAD, 2012). Assim, satisfação no trabalho deriva de uma avaliação feita individualmente a partir dos valores do próprio indivíduo com relação ao trabalho desenvolvido.

Locke afirma ainda que os indivíduos podem vivenciar, num mesmo trabalho, níveis de satisfação e insatisfação diferentes, tendo a satisfação no trabalho como fatores interrelacionados: (1) aos agentes, que agrupam características como: relacionamento entre os chefes, entre colegas e os subordinados, o próprio self do indivíduo, as políticas da empresa e o estilo da gerência; e (2) aos eventos, que representam os aspectos como o trabalho em si, o pagamento, a política de promoção, o reconhecimento verbal e as condições de trabalho. Estes fatores seriam os principais influenciadores tanto para a satisfação quanto para a insatisfação percebida no trabalho. De forma simples, em uma mesma organização pode haver percepções diferentes dos trabalhadores em aspectos relacionados à sua 
atividade, uma vez que nem sempre atribuímos valores iguais a momentos diferentes nas relações com o trabalho.

Contudo, Hackman e Oldham (1976) ressaltam a impossibilidade de dissociar a satisfação no trabalho dos aspectos relacionados às tarefas realizadas. Martinez (2002) corrobora com Locke ao afirmar que, na percepção do trabalhador, a satisfação e a insatisfação fazem parte do mesmo fenômeno onde um mesmo trabalho pode dar a percepção de satisfação e de não satisfação dependendo do momento daquele sujeito.

Segundo Siqueira (2008), a aproximação entre os processos de motivação e a satisfação no trabalho, embasou vários estudos da satisfação do trabalhador. Este fato pode ser ratificado pela influência de diferentes teorias como a teoria de Herzberg, Mausner e Snyderman (1959), a teoria das expectativas de Vromm (1964) e a teoria de Hackman e Porter (1971). Contudo, hoje percebemos ser fundamental separar os construtos de satisfação no trabalho do construto motivação em função de seus impactos no trabalho e no trabalhador (MARTINS \& SANTOS, 2006).

Martins (1984), baseada na definição de Locke, afirmava que o homem usa de sua bagagem individual de crenças e valores para avaliar seu trabalho e essa avaliação resulta num estado emocional que, se for agradável, produz satisfação, e, se for desagradável, leva à insatisfação. Portanto, satisfação no trabalho é uma variável de natureza afetiva e se constitui num processo mental de avaliação das experiências no trabalho que resulta num estado agradável ou desagradável. Esse processo é influenciado pelos vários conteúdos mentais do indivíduo, como crenças, valores, fatores disposicionais, moral e possibilidade de desenvolvimento no trabalho (LEVIN \& STOCKS, 1989; STAW \& ROSS, 1985; STAW, BELL \& CLAUSEN, 1986) e resulta numa tendência que orienta o comportamento. Neste sentido, é uma atitude (FISHBEIN \& AZJEN, 1976).

Para um grupo de teóricos como Vroom (1964), Loffquist e Davis (1969), Orpen (1974), Landy (1978, em Toulson \& Smith, 1994), as reações afetivas dos indivíduos dependem da interação entre eles e seu meio. Para outro grupo, no qual estão Herzberg et al. (1959) e Locke (1976), o indivíduo precisa satisfazer necessidades ou atender a valores para sentir-se satisfeito no trabalho.

Parece, então, haver duas vertentes sobre satisfação no trabalho, que defendem ideias diferentes. Em uma, as características do trabalho são os principais determinantes da satisfação e para a outra, o processamento das informações sociais é o aspecto mais relevante. Apesar de suas divergências, ambas enfatizam o papel dos aspectos situacionais 
que gerariam as atitudes e, portanto, a satisfação no trabalho (PFEFFER \& SALANCIK, 1978).

De acordo com Siqueira (2008), o construto de satisfação no trabalho, a partir da década de 1970, passou a ser entendido como uma atitude com a capacidade preditora dos comportamentos diretamente relacionados ao trabalho. Este enfoque foi fundamental para compreensão do construto e continuidade dos estudos sobre o tema, passando a não ser mais associada a uma organização, e sim a interface trabalho-trabalhador. Brief e Weiss (2002) observaram que as atitudes de cada indivíduo podem influenciar a satisfação no trabalho. Para os autores, os estados afetivos podem provocar impactos positivos ou negativos em diversos resultados pertinentes ao desempenho profissional, como julgamentos, criatividade, comportamento solidário, respostas atitudinais e tomada de decisão.

Indo além, Brief e Weiss afirmam ainda que a forma com que o trabalho afeta os indivíduos e como estes afetam o trabalho em si sofre influência de aspectos como humor, emoções, inveja e ciúme. Satisfação no trabalho decorre das emoções e experiências vividas dentro do contexto laboral e que afetam aspectos da vida do trabalhador. Independente do foco atitudinal ou motivacional dado ao tema é possível se perceber associação entre a satisfação no trabalho e a produtividade do trabalhador.

Diversos estudos foram realizados no Brasil a partir das décadas de 1970 e 1980, e alguns instrumentos foram validados, como os de Nogueira e Pasquali (1981), os de Martins (1984) e Siqueira (1978). O instrumento construído por Martins (1984) avaliava a satisfação no trabalho como sendo bifatorial, relacionando-o diretamente com a teoria dos dois fatores de Herzbert et al (1959), em que os fatores da satisfação aparecem separados da insatisfação. Contudo, Brayfiel e Rothe (1951) publicaram o primeiro instrumento de medida da satisfação no trabalho que aborda aspectos da relação do trabalhador com a atividade tanto de forma positiva quanto negativa como por exemplo os itens (5) eu considero meu trabalho bastante desagradável ou o item (10) estou satisfeito com o meu trabalho por enquanto; ou ainda os itens (2) meu trabalho é como um hobby para mim; ou (6) eu gosto do meu trabalho mais do que o meu tempo de lazer que comparam seu interesse pela trabalho com o lazer e um possível hobby. Este instrumento foi considerado um dos mais utilizados (MARTINS \& SANTOS, 2006).

Siqueira (2008), no início do século XXI, desenvolve um instrumento de avaliação da satisfação voltado para a relação trabalho-trabalhador numa organização de trabalho, contemplando cinco fatores ou dimensões correlacionadas: satisfação com os colegas de 
trabalhos, satisfação com o salário, satisfação com a chefia, satisfação com as promoções e satisfação com a natureza do trabalho. Este instrumento, como a maioria, avalia satisfação no trabalho no ambiente organizacional, portanto, na vinculação formal de trabalho.

Com relação ao trabalho informal não existem instrumentos para medir o nível de satisfação. Embora o trabalho se caracterize como uma forma diferenciada de trabalho sem chefia, promoção ou salário fixo, têm uma relação de trabalho formada diretamente com os usuários dos seus serviços e com a sociedade. Esta relação, associada com a renda gerada a partir da atividade em si pode implicar em satisfação ou não com o trabalho. Conforme o IBGE (2003) o setor informal é constituído por trabalhadores sem carteira assinada, empregadores com até 5 empregados sem carteira assinada e os trabalhadores por conta própria (SASAKI, 2009).

Pastore (2004) difere os trabalhadores informais em dois grupos distintos: (1) empregados sem registro em carteira, sem remuneração predefinida e trabalhadores domésticos (hoje com legislação específica); e (2) trabalhadores por conta própria e empregadores informais, categorias distintas que exigem um tratamento diferenciado quanto às normas de relação do trabalho. Pastore (2005), analisando o crescimento do trabalho informal, atribui ao desenho de uma nova forma de trabalho, com aumento da escolaridade e influência dos avanços tecnológicos exigindo novas formas de conhecimento muitas vezes não vinculadas à qualificação formal.

Com relação ao trabalho em si, Codo $(1994,2002,2004,2006)$ afirma que o trabalho não pode ser discutido somente a partir de seu vínculo, processo ou pela objetividade da ação em si, mas também da subjetividade nela existente. Para o autor, os trabalhadores ao se integrarem ao circuito produtivo deixam sua marca. O gesto, como significado da atividade humana e formador de seu conteúdo, a tarefa como ação, o ambiente como o espaço em que a ação-trabalho ocorre e as condições objetivas do trabalho como carga de trabalho, rotinas e normas, além das condições subjetivas do trabalho como relacionamento com colegas, chefias e clientela, percepção de suporte social e afetivo, conflito trabalho e família compõem de forma indissociável a relação trabalho-trabalhador e devem ser consideradas em qualquer avaliação desta relação.

Os diversos elementos que compõem a tarefa pressupõem relações complexas que irão influenciar não só trabalho em si, mas nas percepções acerca de si mesmo e da tarefa realizada. As relações de troca existentes pelo reconhecimento de seu trabalho e pela remuneração passam a representar para o trabalhador a forma de reapropriação da 
sociedade, do consumo, da cultura e do lazer. Com base nesta concepção que aborda a relação trabalho-trabalhador através dos aspectos objetivos e subjetivos do trabalho e considerando a não existência de uma escala para avaliação da satisfação no trabalho voltada para trabalhadores informais e para trabalhadores por conta própria, o objetivo deste estudo é construir e validar a Escala de Satisfação no Trabalho para Trabalhadores Informais (EST-TI).

\section{MATERIAIS E METODOS}

O presente estudo foi avaliado pelo Comitê de Ética e Pesquisas com Seres Humanos da Universidade Salgado de Oliveira (UNIVERSO), e foi aprovado com o parecer $\mathrm{n}^{\circ}$ 3.236.147. A EST-TI foi construída em três etapas subsequentes e complementares antes de sua aplicação junto aos trabalhadores informais. Na primeira etapa foram definidas as dimensões teóricas a partir da literatura, sendo elas: relação trabalho-trabalhador; remuneração; relação com clientes e com os pares; segurança e previdência social. Estas dimensões serviram de base para a construção dos itens. A segunda etapa consistiu em submeter os itens construídos à análise teórica, visando a clareza e coerência dos mesmos.

A terceira etapa constou na aplicação dos itens da escala à trabalhadores informais de diferentes áreas de atuação para fatoração posterior. Utilizou-se o software SPSS versão 22 para analisar estatisticamente os itens propostos no instrumento através de uma análise fatorial exploratória. Foram extraídos os principais fatores através do método de fatoração dos eixos principais, bem como as correlações entre os itens, analise de carga fatorial e o alfa de Cronbach.

\section{Elaboração e Análise Semântica dos Itens}

Os itens foram elaborados a partir das formulações teóricas tendo por base as concepções sobre trabalho de Codo (1994, 2002, 2004, 2006), levando em consideração às dimensões objetivas e subjetivas do trabalho, bem como as relações existentes e a percepção de satisfação no trabalho. De forma complementar, a teoria dos agentes e eventos de Locke (1976) e a Escala de Satisfação no Trabalho organizacional desenvolvida e validada por Siqueira (2008), mesmo com abordagens distintas, contribuíram para a formulação dos itens. Para a construção dos itens levou-se em consideração os critérios estabelecidos por Pasquali (2010), como objetividade, simplicidade, clareza, relevância, precisão e variedade. 
A análise semântica foi realizada a fim de verificar se todos os itens estavam claros e precisos de forma a permitir plena compreensão pela clientela alvo. Foram utilizados três juízes professores universitários na área de psicologia do trabalho. Os juízes realizaram o julgamento considerando o conteúdo abordado, clareza e compreensão, e relevância de cada item. $\mathrm{O}$ trabalho dos juízes foi individual e pré-estabelecido a concordância de pelo menos dois juízes para aceitação do item.

\section{Análise das Evidências de Validade da Escala}

\section{a. Participantes}

Participaram do estudo 328 trabalhadores informais pertencentes as diversas áreas de atuação, a decisão de compor a amostra com participantes pertencentes aos diversos campos de atuação do trabalhador informal deveu-se ao propósito de validar um questionário de caráter genérico, aplicável a diferentes condições de trabalho e não apenas a um segmento específico. Os participantes, a partir de suas atividades, foram classificados em treze grupos de atuação: (1) construção civil (8,7\%); (2) comércio ambulante $(2,8 \%)$; (3) balé, dança, artes plásticas e artesanato (5,6\%); (4) estética e beleza (10,6\%); (5) costura e confecção (3,1\%); (6) vendas e consultoria de vendas (37,6\%); (7) serviços administrativos e de atendimento (5\%); (8) serviços técnicos (8,4\%); (9) bolos, doces e salgados (4\%); (10) serviços domésticos (3,1\%); (11) serviços especializados $(3,1 \%) ;(12)$ pesca $(3,7 \%)$; e (13) motorista $(4,3 \%)$.

Do público pesquisado, 52\% eram do sexo feminino, 20,6\% tinham até 24 anos de idade, $20,8 \%$ de 25 a 34 anos, $22,4 \%$ de 35 a 44 anos, $24,9 \%$ de 45 a 55 e 11,3\% acima de 55 anos. Em relação à escolaridade, $59 \%$ possuíam o ensino médio completo, 22,2\% algum tipo de graduação superior e 18,7 o ensino fundamental. Afirmaram ser casados ou estarem em união estável $54,8 \%$, sendo mais $37,1 \%$ solteiros e $8,1 \%$ divorciados ou viúvos. A maioria dos pesquisados $(57,8 \%)$ possuíam quatro anos ou mais de tempo na informalidade.

$\mathrm{Na}$ seleção dos participantes, não foram feitas distinções de sexo, raça, condição socioeconômica, tipo de trabalho realizado e tempo de informalidade. Como critério de inclusão os participantes deveriam ter mais de 18 anos e ter o trabalho informal como principal fonte de renda pessoal. Foram excluídos do estudo os trabalhadores informais por 
ocasião, ou seja, aqueles que realizam trabalhos informais de forma temporária ou como segunda fonte de renda.

\section{b. Instrumento}

O instrumento inicialmente era composto por 79 itens pertinentes a uma das seis dimensões pré-definidas. Os itens foram organizados aleatoriamente e utilizados em uma escala do tipo Likert de 7 pontos, sendo em um extremo o 1 como discordo totalmente e crescendo gradativamente o grau de concordância o 7 como concordo totalmente. Além dos itens referentes à escala, o instrumento incluía dados sociodemográficos referentes à escolaridade, sexo, idade, estado civil, tempo na informalidade e atividade desempenhada.

\section{c. Procedimentos de coleta de dados}

A coleta de dados foi realizada com trabalhadores informais que atuam no Leste Metropolitano do Estado do Rio de Janeiro (Niterói, São Gonçalo, Maricá, Itaboraí, Magé, Rio Bonito, Cachoeiras de Macacu, Tanguá e Magé) no período compreendido entre março e outubro de 2017. Os trabalhadores foram chamados a participar de forma voluntária, assinaram o Termo de Consentimento Livre e Esclarecido e responderam o instrumento.

O tempo médio de resposta aos instrumentos foi de 15 minutos. A coleta de dados foi realizada pelo pesquisador e pelos alunos de graduação em Psicologia.

\section{d. Análise dos dados}

Foi realizada análise estatística descritiva dos dados coletados e verificada a viabilidade da análise fatorial exploratória. Identificou-se a presença de missings (dados ausentes) num percentual muito baixo, não comprometendo a análise dos dados de dados, sendo procedida a retirada dos casos com missings, tendo em vista a adequação desse tipo de procedimento diante da baixa porcentagem de missings (TABACHNICK \& FIDEL, 2001). O pressuposto estatístico fatorabilidade foi investigado pelo KMO (Kaiser-MeyerOlkin) e a esfericidade pelo Teste de Esfericidade de Bartlett.

Para validação do instrumento, foi conduzida uma análise fatorial pelo método de fatoração dos eixos principais, rotação oblimin direto. Procedeu-se ainda à análise de consistência interna pelo alfa de Cronbach. Foi utilizado o programa SPSS (Statistical 
Package for the Social Sciences, versão 22). Como critério de retenção de fatores, foi utilizada a análise da variância total explicada e o gráfico de scree plot. A dimensionalidade do instrumento também foi investigada por uma análise paralela, realizada através do software Factor em sua versão 7.0.

\section{RESULTADOS}

Para a condução da análise fatorial foi realizada uma inspeção inicial da matriz de correlações (Correlation Matrix) e verificadas as possibilidades de fatorabilidade. A matriz foi considerada fatorável, já que a medida KMO (Kaiser- Meyer-Olkin) foi alto $(0,834)$ e o Teste de Esfericidade de Bartlett's (qui-quadrado aproximado: 11400,402; nível de significância de 0,0001 ) indicou que a matriz correlacional não era uma matriz identidade.

Com o intuito de avaliar o número de fatores a serem extraídos, observou-se a variância total explicada por cada fator, conforme apresentado nos resultados da análise fatorial exploratória. Ao serem comparados os autovalores e a variância explicada, foram retidos quatro fatores nos quais os autovalores eram maiores do que 3,0. Além disso, foi realizada a análise paralela que indicou a retenção de 4 fatores.

Em seguida prosseguiu-se com a análise fatorial exploratória utilizando-se 0 Softwares SPSS em sua versão 22. A análise foi realizada utilizando o método de rotação oblíqua oblimin direto, onde foram extraídos quatro fatores. $O$ fator 1 foi denominado relação trabalho-trabalhador-cliente, sendo composto por 15 itens. 0 fator 2 , intitulado segurança e vínculo, agregou 8 itens. O fator 3 foi chamado de rendimento e qualidade de vida, contemplando um total de 11 itens. O fator 4, denominado de desenvolvimento pessoal e profissional, é composto de 5 itens. A análise dos quatro fatores separadamente revelou os seguintes valores do alfa de Cronbach: Fator $1 \mathrm{com}$ alfa de 0,93, Fator 2 com alfa de 0,84 , Fator $3 \mathrm{com}$ alfa de 0,90 e Fator $4 \mathrm{com}$ alfa de 0,86. Foram retirados os itens $1,4,5,8,9,10,11,12,13,16,17,19,20,21,22,23,27,29,30,33,36,37,38,42,44, .45$, $46,49,50,52,55,56,61,63,65,67,70,75,77$ e 79 por apresentarem carga fatorial abaixo de 0,50. O resultado da análise fatorial exploratória está na Tabela 1.

Tabela 1. Análise Fatorial Exploratória

\begin{tabular}{lcc}
\hline & Fator \\
\hline & 1 & 2 \\
\hline ITEM 69 Estou satisfeito com a maneira que meus clientes me tratam &, 92 \\
ITEM 76 Estou satisfeito com a maneira que me relaciono com meus clientes &, 89 \\
ITEM 71 Estou satisfeito com o entendimento entre meus clientes e eu &, 83 \\
\hline
\end{tabular}


ITEM 66 Estou satisfeito com a confiança que eu posso ter em meus clientes

ITEM 54 Estou satisfeito com a confiança que meus clientes demonstram pelo meu trabalho

ITEM 78 Estou satisfeito com o interesse dos meus clientes pelo meu trabalho $\quad$,76

ITEM 62 Estou satisfeito com a amizade que eu tenho pelos meus clientes $\quad$,76

ITEM 58 Estou satisfeito com a confiança que meus clientes têm em mim $\quad$,75

ITEM 73 Estou satisfeito com o que os clientes dizem do meu trabalho ,75

ITEM 35 Estou satisfeito com a maneira que os clientes me tratam 62

ITEM 26 No meu trabalho tenho que agradar meus clientes ,61

ITEM 43 Preciso da colaboração de meus clientes para me manter trabalhando , 58

ITEM 32 Gosto das tarefas que desenvolvo no meu trabalho

ITEM 47 Gosto do meu trabalho

ITEM 64 Gosto de fazer o trabalho que faço $\quad, 50$

ITEM 74 Tenho pensado em procurar um trabalho com mais estabilidade $\quad$ 69

ITEM $31 \mathrm{~A}$ falta de acesso ao seguro desemprego é uma preocupação $\quad$ 67

ITEM 28 Me sinto inseguro sem carteira assinada $\quad$,66

ITEM 18 Me assusta não ter carteira assinada $\quad$,64

ITEM 6 Tenho pensado em procurar emprego de carteira assinada , 63

ITEM 68 Tenho pensado em mudar de trabalho ,62

ITEM 59 Com este trabalho me sinto inseguro com relação ao meu futuro $\quad$,56

ITEM 7 Tenho pensado em procurar um trabalho que me exponha menos a $\quad$ 54

situações de riscos e de inseguranças

ITEM 53 Estou satisfeito com o que eu recebo pelo meu trabalho

ITEM 48 O que eu ganho com o meu trabalho permite que eu viva

satisfatoriamente

profissional

ITEM 2 Estou satisfeito com o rendimento financeiro que posso obter no meu trabalho

ITEM 72 Gosto da qualidade de vida que meu trabalho me proporciona

ITEM 57 Estou satisfeito com o meu rendimento comparado aos meus esforços no trabalho

ITEM 14 O que eu ganho (dinheiro) no meu trabalho está de acordo com o meu esforço

ITEM 39 Meu trabalho permite que eu viva adequadamente

ITEM 51 Percebo que trabalhadores com carteira assinada têm inveja do meu trabalho

ITEM 15 Meu trabalho permite crescimento profissional 
Os quatro fatores extraídos da análise dos eixos principais revelaram-se válidos, em decorrência das excelentes cargas fatoriais de seus itens e da coerência teórica existente. Foram encontradas cargas fatoriais variando de 0,504 a 0,920, sendo que valores acima de 0,50 são considerados necessários para a significância prática (Hair et al., 2009). A consistência interna dos fatores $1,2,3$ e 4 foram satisfatórias, respectivamente $\alpha=0,93, \alpha$ $=0,85, \alpha=0,91$ e $\alpha=0,87$.

\section{DISCUSSAOO}

Duas perspectivas merecem nortear a discussão acerca dos resultados do estudo: o enfoque teórico e os pressupostos da psicometria. Em relação à teoria, tem sido sustentado por Codo $(1994,2002,2004,2006)$ que a subjetividade e a objetividade do trabalho têm impacto na relação trabalho-trabalhador e, consequentemente, em sua satisfação. Esta visão considera que as atitudes e às situações a que são expostos influenciam a percepção dos trabalhadores em relação a seu trabalho, influenciando nos níveis de satisfação ou insatisfação dos mesmos em dado momento. De fato, a proposta de considerar os aspectos objetivos e subjetivos do trabalho facilitou a compreensão psicológica do fenômeno da satisfação e permitiu elaborar medidas para aferi-lo.

Em relação aos pressupostos psicométricos, ressalta-se o fato de que, entre os diversos instrumentos que se destinam a medir satisfação no trabalho, todos são voltados a realizar esta análise apenas com foco no trabalho formal. Importante notar, contudo, que a EST-TI foi construída e validada para um grupo específico de trabalhadores, os do trabalho informal, contexto em que se torna mais fácil identificar diferenças entre os construtos de satisfação no trabalho formal e informal.

A identificação de quatro dimensões correlacionadas entre si, permite afirmar que os resultados obtidos neste estudo confirmam a teoria multidimensional, como o estudo de (SIQUEIRA, 2008). A partir de um conjunto inicial de 79 itens, foi utilizado o método de extração de Fatoração de Eixos Principais e rotação Oblimin em três séries consecutivas de análises com o objetivo de redução da ordem da matriz de intercorrelações. Após as análises foram identificados 4 fatores compostos por 39 itens que, com base em seus 
conteúdos, foram reunidos em quatro dimensões amplas: relação trabalho-trabalhador, rendimento e qualidade de vida, segurança e vínculo e desenvolvimento pessoal e profissional.

Os resultados obtidos permitiram concluir que os procedimentos de análise realizados neste estudo, identificaram de maneira adequada a estrutura empírica do construto satisfação no trabalho. Os procedimentos de análise de dados foram criteriosos em seus aspectos, em especial na escolha dos métodos de extração e de rotação. Contudo, vale ressaltar que Nunnaly e Bernstein (1995) afirmam que as soluções oblíquas e ortogonais conduzem, provavelmente, às mesmas conclusões. Este fato pode ser confirmado pela realização de uma análise fatorial com rotação Oblimin, não relatada no texto, em que o banco de dados analisado apontou os mesmos fatores. Destaca-se, ainda, que a solução fatorial identificada permitiu a explicação de parte considerável da variância das dimensões do construto de satisfação no trabalho.

Os resultados das análises fatoriais deste estudo demonstram que a EST-TI é passível de fatoração e que se constitui em uma medida válida e com indicativos de precisão. A análise de confiabilidade apresentou alfa de 0,901 . Todos os 39 itens possuem cargas fatoriais acima de 0,50 . Foram encontrados quatro fatores teoricamente consistentes e psicométricamente com alfas superiores a 0,85 . Os quatro fatores correspondem respectivamente: relação trabalho-trabalhador-cliente (Fator 1), segurança e o vínculo (Fator 2), rendimento e qualidade de vida (Fator 3) e desenvolvimento pessoal e profissional (Fator 4).

No Fator 1, com quatorze itens, agrupam três itens de representação do trabalho em si e onze itens de representação da relação do trabalhador informal com o trabalho e com seus clientes. No Fator 2, com oito itens, agrupam cinco itens relacionados a percepção de segurança no trabalho e três relacionados ao vínculo com o trabalho. A questão de segurança e vínculo no trabalho, muitas vezes coloca em oposição trabalhadores formais e trabalhadores informais. Aqui a questão está sendo discutida nos oito itens, permitindo compreender se, de fato, os trabalhadores informais sofrem com a perspectiva de insegurança da informalidade.

No Fator 3, de onze itens, foram agrupados sete itens referentes à remuneração e quatro itens referentes à qualidade de vida do trabalhador informal. Estudo desenvolvido por Sassaki e Vasques-Menezes (2009) mostra que os trabalhadores informais afirmam obter uma renda superior a que teriam em igual atividade como trabalhador formal. Contudo, este ainda é um aspecto contraditório quando se compara trabalhadores formais 
e informais. Este fator aborda exatamente este ponto e será de grande valia para este entendimento.

No Fator 4, de cinco itens, três itens se agruparam referentes ao desenvolvimento pessoal e dois itens ao desenvolvimento profissional dos trabalhadores informais. Desenvolvimento pessoal e profissional é apontado com frequência como dos problemas que os trabalhadores informais enfrentam. Estes fatores são assim definidos:

- Fator 1 - Relação trabalho-trabalhador-cliente - percepção de interesse e adequação às atividades realizadas e relacionamento com os clientes dos serviços prestados.

- Fator 2 - Segurança e Vínculo -percepção de segurança e estabilidade proporcionada pelo trabalho e perspectivas de futuro.

- Fator 3 - Rendimento e Qualidade de Vida - percepção dos trabalhadores acerca da adequação ou não dos rendimentos recebidos em relação ao trabalho realizado e as perspectivas de qualidade de vida.

- Fator 4 - Desenvolvimento Pessoal e Profissional - percepção do trabalhador sobre a possibilidade de crescimento profissional e pessoal a partir do trabalho.

Contudo, grande parte das escalas psicométricamente válidas e legítimas levam em consideração realidades da organização institucional do trabalho sendo direcionadas para o trabalhador formal. A EST-TI, com item que correspondem aos aspectos objetivos e subjetivos do trabalho, está em consonância ao modelo teórico previamente definido e aponta para a relação trabalho-trabalhador informal.

A importância das dimensões do construto de satisfação no trabalho como antecedentes e como consequentes de outras variáveis de múltiplos níveis tem sido demonstrada, no caso dos trabalhadores formais, através de diversos estudos (ALBUQUERQUE, 2003; ANTLOGA, 2003; MACHADO, 1997; MENDES, 1999; MORRONE, 2001; MOTA, 2002; PASCHOAL, 2003; PEREIRA, 2003; RESENDE, 2003; SILVA, 1999; STACCIARINI, 1999).

No caso dos trabalhadores informais, esta relação é pouco discutida em função da falta de instrumentos apropriados. Desta forma, a discussão e validação de uma escala de satisfação no trabalho voltada especificamente para os trabalhadores informais, a EST-TI , com características psicométricas adequadas, e dimensões semelhantes às escalas de satisfação no trabalho para as organizações institucionais, pode ser bastante útil para realização de diagnósticos e investigações futuras e representa um ganho significativo pois 
fornecerá aos pesquisadores um instrumento específico para o estudo em trabalhadores informais, com características e itens adequados a este tipo de trabalhador.

\section{CONSIDERAÇOES FINAIS}

O presente artigo contribui ao apresentar evidências iniciais de validade da EST-TI. Os resultados indicam que esta escala pode se constituir como uma ferramenta útil na avaliação dos índices de satisfação no trabalho em trabalhadores informais. O uso da ESTTI pode ser vantajoso por permitir que as experiências de satisfação no trabalho dos profissionais informais para realizar suas atividades laborais sejam avaliadas por meio de uma escala breve. A possibilidade de avaliar a satisfação no trabalho dos profissionais em seu cotidiano permite mapear o desenvolvimento dos processos de trabalho e contexto laboral. Estas informações podem ser utilizadas como evidências para o desenvolvimento de intervenções efetivas visando melhorias nas condições de trabalho.

A principal limitação deste estudo envolve a utilização de uma amostra por conveniência situado na região do Leste Metropolitano do Estado do Rio de Janeiro, restringindo a representatividade da amostra em relação aos trabalhadores informais. Sugere-se o desenvolvimento de estudos complementares em outras amostras maiores (grupos de trabalhadores de segmentos específicos) para confirmação dos achados, bem como a condução de análises fatoriais confirmatórias.

De maneira semelhante, a realização de estudos discriminantes entre os construtos de satisfação no trabalho e outros como envolvimento com o trabalho, centralidade do trabalho e comprometimento organizacional afetivo poderiam trazer contribuições interessantes para a compreensão dos impactos do construto. Ressalta-se que são escassos os estudos sobre essa temática no Brasil e espera-se, com a disponibilização dessa ferramenta, contribuir para o aumento de pesquisas na área da satisfação no trabalho voltadas para o trabalhador informal.

\section{REFERÉNCIAS}

ALBUQUERQUE, A. S. (2003). Bem-estar subjetivo e sua relação com personalidade, coping, suporte social, satisfação conjugal e satisfação no trabalho. (Tese de Doutorado não-publicada). Instituto de Psicologia, Universidade de Brasília, DF.

ANTLOGA, C. S. X. (2003). Estilo de contato da organização como funcionário e prazer e sofrimento no trabalho: estudo de caso em uma empresa de material de construção no Distrito Federal (Dissertação de Mestrado). Brasília: Universidade de Brasília. Instituto de Psicologia. 
BACHA, A. M., GRASSIOTTO, O. DA R., GONÇALVES, S. P., HIGA, R., FONSECHICARVASAN, G. A., MACHADO, H. DA C. \& CACIQUE, D. B. (2015). Satisfação no trabalho da equipe de enfermagem em um hospital universitário. Revista Brasileira de Enfermagem, 68(6), 1130-1138.

BRANDÃO, I. DE F., LIMA, L. C. DE, CABRAL, A. C. DE A., SANDRA MARIA DOS SANTOS, S. M. DOS, PESSOA, M. N. M. (2014). Satisfação no serviço público: um estudo na Superintendência Regional do Trabalho e Emprego no Ceará. READ Revista eletrônica de administração. Porto Alegre, 20(1), 90-113.

BRAYFIELD, A. H. \& ROTHE, H. F. (1951). An index of job satisfaction. Journal of Applied Psychology, 35, 307-311.

BRIEF, A. P. \& WEISS, H. M. (2002). Organizational behavior: Affect in the workplace. Annual Review of Psychology, 53, 279-307

CARLOTTO, M. S. \& CÂMARA, S. G. (2008). Propriedades psicométricas do Questionário de Satisfação no Trabalho (S20/23). PsicoUSF, 13(2), 203-210.

CODO, R.; FONSECA, G. F. (2004). Em busca do significado do trabalho: relato de um estudo qualitativo entre executivos. Revista Brasileira de Gestão de Negócios, 6 (14), 7 18.

CODO, W. (1994). O que é alienação. 10ª ed. São Paulo: Ed. Brasiliense. (Coleção Primeiros Passos).

CODO, W. (2002). Educação: carinho e trabalho. 3aㅗ ed. Petrópolis: Rio de Janeiro: Vozes.

CODO, W. (2004). Prototeoria: síndrome do trabalho vazio. In: Codo, W. (Org.). O trabalho enlouquece?: um encontro entre a clínica e o trabalho. Petrópolis: Vozes.

CODO, W. (2006). Ação dos psicólogos nos sindicatos: trabalho, alienação e transformação social (1987). In: Codo, W. (Org.). Por uma psicologia do trabalho. São Paulo: Casa do Psicólogo. 7-50.

COELHO, F. A. JR. \& FAIAD, C. (2012). Evidências de validade da Escala de Satisfação no trabalho. Avaliação Psicológica, 11(1), 111-121.

FISHBEIN, M. \& AZJEN, I. (1976). Belief, attitude, intention and behavior: An introduction to theory and research. London: Addison-Wesley.

HACKMAN, J. R. \& OLDHAM, G. R. (1976). Motivation through the design of work: Test of a theory. SOM: Yale University.

HACKMAN, R.; PORTER, L. W. (1971). Predicciones de la eficiencia en el trabajo por la teoria de las expectativas. In: Fleishman, E. A.; BASS, A. R. (Org.). Estudios de psicologia industrial y del personal. México: Trillas.

HERZBERG, F., MAUSNER, B. \& SNYDERMAN, B. (1959). The motivation to work. New York: Wiley.

INSTITUTO BRASILEIRO DE GEOGRAFIA E ESTATÍSTICA (IBGE). (2004). Pesquisa Nacional de Amostra por Domicílio (PNAD). Rio de Janeiro, IBGE. 
LEVIN, I. \& STOCKS, J. P. (1989). Dispositional approach to the job satisfaction: Role of negative affectivity. Journal of Applied Psychology, 74(5), 752-758.

LOCKE, E. A. (1976). The nature and causes of job satisfaction. In Dunnette, M. D. (Org.). Handbook of industrial and organizational psychology. Chicago: Rand McNally College Publishing Company, 1297-1350.

LOFFQUIST, L. H. \& DAVIS, R. V. (1969). Adjustment to work. New York: Appleton Century Crofts.

MACHADO, S. S. (1997). Fatores relacionados ao estresse ocupacional em ambientes de risco (Dissertação de Mestrado). Brasília: Universidade de Brasília. Instituto de Psicologia.

MARQUES, A. L., BORGES, R. \& REIS, I. DO C. (2016). Mudança organizacional e satisfação no trabalho: um estudo com servidores públicos do estado de Minas Gerais. Revista de Administração Pública, 50(1), 41-58.

MARTINEZ, M. C. (2002). As relações entre a satisfação com aspectos psicossociais no trabalho e a saúde do trabalhador. Dissertação de Mestrado, Universidade de São Paulo, São Paulo, SP, Brasil.

MARTINS, M. D. C. F. (1984) Satisfação no trabalho: elaboração de instrumento e variáveis que afetam a satisfação. (Dissertação de Mestrado), Universidade de Brasília, Brasília, DF, Brasil.

MARTINS, M. D. C. F., \& SANTOS, G. E. (2006). Adaptação e validação de construto da Escala de Satisfação no Trabalho. Psico-USF, 11(2), 195-205.

MENDES, A. M. (1999). Valores e vivências de prazer. sofrimento no contexto organizacional (Tese de Doutorado). Brasília: Universidade de Brasília. Instituto de Psicologia.

MORRONE, C. F. (2001). Só para não ficar desempregado. Ressignificando o sofrimento psíquico no trabalho: estudo com trabalhadores em atividades informais (Dissertação de Mestrado). Brasília: Universidade de Brasília. Instituto de Psicologia.

MOTA, L. M. O. (2002) Treinamento e prazer. Sofrimento psíquico no trabalho (Dissertação de Mestrado). Brasília: Universidade de Brasília. Instituto de Psicologia.

NOGUEIRA, P. R. \& PASQUALI, L. (1981). Satisfação no trabalho - construção de um instrumento. Arquivos Brasileiros de Psicologia, 33, 3-18.

NUNNALY, J. C. \& BERNSTEIN, I. J. (1995). Teoría Psicométrica. Buenos Aires: McGraw Hill.

ORPEN, C. (1974). A cognitive consistency approach to job satisfaction. Psychological Reports, 36, 239-245.

PASCHOAL, T. (2003) Relação dos valores do trabalho e da interferência famíliatrabalho com estresse ocupacional (Dissertação de Mestrado). Brasília: Universidade de Brasília. Instituto de Psicologia. 
PASQUALI, L. (2010). Instrumentação psicológica: fundamentos e práticas. Porto Alegre: Artmed.

PASTORE, J. (2004). Informalidade: estragos e soluções. Recuperado em 25 de agosto de 2018 de http://www.josepartore.com.br/ti/index.htm.

PARTORE, J. (2005). Perspectiva e Problemas de Emprego no Brasil. Seminário Brasil Canadá: Desafios para a criação de empregos, patrocinando pelo CEBRI. Brasília, recuperado em 10 de setembro de 2018 de http://www.cebri.org.br/pdf/232_pdf.pdf.

PFEFFER, J. AND SALANCIK, G. R. (1978), The External Control of Organizations: A Resource Dependence Perspective, Harper \& Row, New York.

PEREIRA, J. A. S. (2003). Vivências de prazer e sofrimento na atividade gerencial em empresa estratégica: o impacto dos valores (Dissertação de Mestrado). Brasília: Universidade de Brasília. Instituto de Psicologia.

RESENDE, S. (2003). Vivências de prazer e sofrimento no trabalho bancário: o impacto dos valores individuais e variáveis Adaptação e validação de construto da Escala de Satisfação no Trabalho. Psico-USF. 11 (2), 195-205.

SASAKI, M. A. (2009). Trabalho Informal: escolha ou escassez de empregos. Estudo sobre o perfil dos trabalhadores por conta própria. Dissertação de Mestrado em Psicologia Social e das Organizações, Brasília: Universidade de Brasília.

SASAKI, M. A. \& VASQUES-MENEZES, I. (2012). Trabalhador informal e Previdência Social: o caso dos trabalhadores por conta própria de Brasília-DF. Política \& Sociedade, (11)21.

SILVA, A. V. (1999). Comportamento de motoristas de ônibus: itinerário urbano, estressores ocupacionais e estratégias de enfrentamento (Dissertação de Mestrado). Brasília: Universidade de Brasília. Instituto de Psicologia.

SIQUEIRA, M. M. M. (1978). Satisfação no trabalho. Dissertação de Mestrado, Universidade de Brasília, Brasília, DF, Brasil.

SIQUEIRA, M. M. M. (1995). Antecedentes de comportamentos de cidadania organizacional: análise de um modelo pós-cognitivo. Tese de Doutorado, Universidade de Brasília, Brasília, DF, Brasil.

SIQUEIRA, M. M. M. (2008). Satisfação no trabalho. In Siqueira, M. M. M. (Org.), Medidas do comportamento organizacional: ferramentas de diagnóstico e gestão. Porto Alegre: Artmed, 265-274.

SOUZA, A. C. DE., MILANI, D. \& ALEXANDRE, N. M. C. (2015). Adaptação cultural de um instrumento para avaliar a satisfação no trabalho. Revista Brasileira de Saúde Ocupacional, São Paulo: 40(132), 219-227.

STACCIARINI. J. M. R. (1999). Estresse ocupacional, estilos de pensamento e coping na satisfação, mal-estar físico e psicológico dos enfermeiros (Tese de Doutorado). Brasília: Universidade de Brasília. Instituto de Psicologia.

STAW, B. M. \& ROSS, J. (1985). Stability in the midst of change: A dispositional approach to job attitudes. Journal of Applied Psychology, 70, 469-480. 
STAW, B. M., BELL, N. E. \& CLAUSEN, J. A. (1986). The dispositional approach to job attitudes: A Lifetime Longitudinal Test. Administrative Science Quarterly, 31, 56-77.

TABACHNICK, B. G. \& FIDEL, L. S. (2001). Using multivariate statistics. San Francisco: Allyn \& Bacon.

TOULSON, P. \& SMITH, M. (1994). The relationship between organizational climate and employee perceptions of personnel management practices. Public Personnel Management, 23(3), 453-468.

VROMM, V. H. (1964). Work and motivation. New York: Wiley. 